\title{
Il ruolo del sistema nervoso vegetativo (SNV) nell'ipotensione in dialisi
}

\author{
V. Savica ${ }^{1}$, G. Bellinghieri ${ }^{1}$, G. Vita ${ }^{2}$, R. Musolino $^{2}$ \\ ${ }^{1}$ Cattedra di Nefrologia, ${ }^{2}$ Istituto di Scienze Neurologiche e Neurochirurgiche \\ Università di Messina, Messina
}

elle specie più evolute, uomo compreso, il Sistema Nervoso Vegetativo occupa un ruolo importante nel controllo della pressione arteriosa su cui agisce attraverso la via parasimpatica e la via simpatica mediante i messaggi inviati dai centri bulbari e soprabulbari (Figg. 1, 2).

L'attivazione della via parasimpatica determina la liberazione di acetilcolina a livello dei nodi cardiaci producendo una azione cronotropa negativa mentre l'attivazione della via simpatica mediante la liberazione di noradrenalina induce una

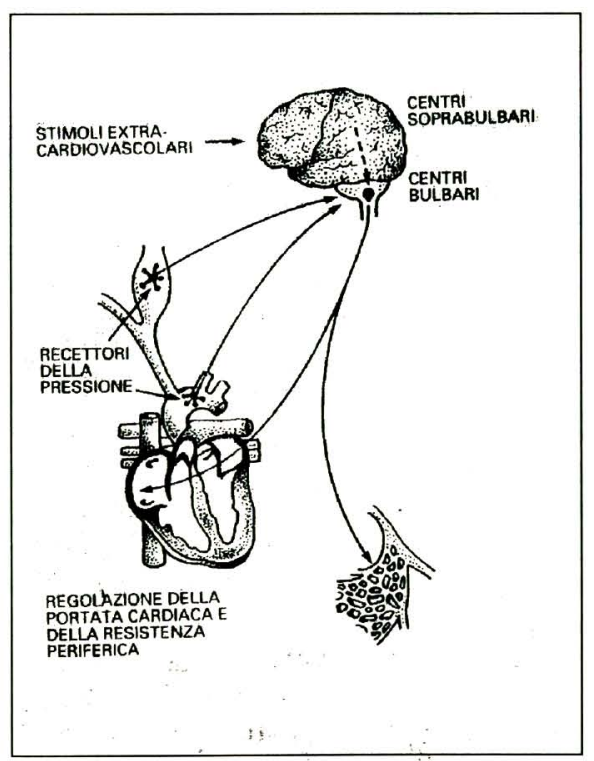

Fig. 1 azione cronotropa e inotropa positiva (Figg. 3, 4). Se prevarranno i recettori adrenergici dei vasi viscerali e cutanei si avrà vasocostrizione mentre se prevarranno i recettori $\beta$-2-adrenergici si avrà vasodilatazione (Fig. 5).

Tutto ciò si traduce in risposta emodinamica che non sarà adeguata se a carico del SNV si realizzano anomalie.
Il SNV è più efficace nel contrastare l'ipotensione arteriosa che l'ipertensione arteriosa. Esso è stato chiamato spesso in causa, nel paziente uremico, specie emodializzato, allorché questi presentava in corso di dialisi episodi ipotensivi intradialitici sintomatici che condizionavano la conduzione del trattamento stesso.

Pertanto molta ateznione è stata rivolta



Fig. 2 - Fattori coinvolti NZL controllo della pressione arteriosa. 


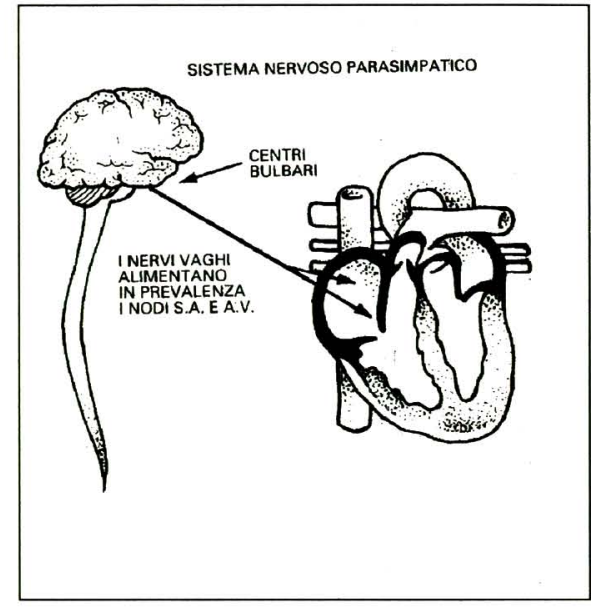

Fig. 3

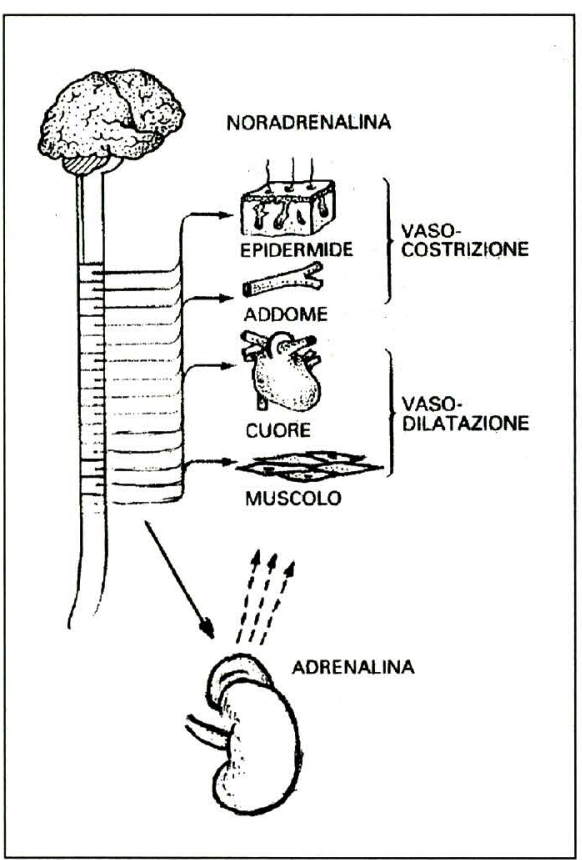

Fig. 5 - Innervazione del sistema simpatico.

allo studio dello stato del SNV nell'uremico e al controllo di questo esercitato sul sistema cardiovascolare. Tale studio nell'uremia, come in altre condizioni patologiche, si basa sulla esecuzione di una batteria di test incruenti, semplici, riproducibili ed attendibili quali lo studio della variabilità dell'intervallo $R-R$ a riposo e durante respirazione profonda, la manovra di Valsalva, la variazione ortostatica della frequenza cardiaca (FC) e della pressione arteriosa (PA) e la contrazione muscolare isometrica (1).

Altri test vengono usati meno comunemente e comunque per studiare in dettaglio alcuni aspetti, quali quelli che valutano la risposta della FC e della PA al

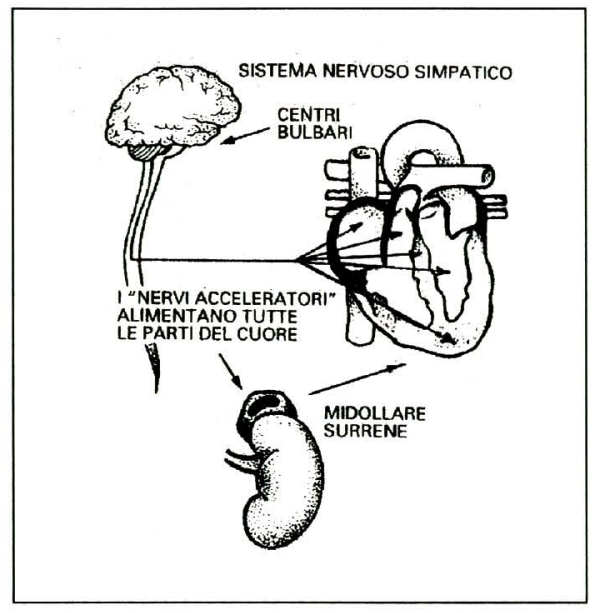

Fig. 4

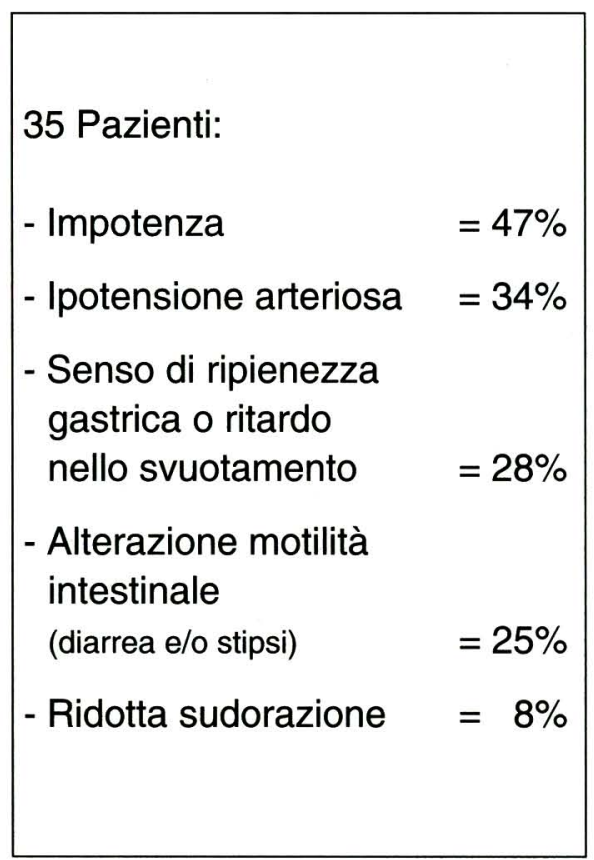

Fig. 6 - Nostra casistica.

calcolo mentale, all'esposizione al rumore intenso, all'immersione della mano in acqua ghiacciata, all'immersione del volto in acqua fredda, al collare pneumatico, alla pressione negativa esercitata sulla parte inferiore del corpo.

Vengono utilizzati inoltre test farmacologici quali quello all'atropina, al propanololo, alla noradrenalina, alla clonidina e il dosaggio delle catecolamine plasmatiche basali e dopo stimolo.

È opinione ormai condivisa da tutti che una compromissione del controllo vegetativo sia da ritenersi provata quando almeno due test, all'interno di una batteria di 4-6 test, risultino patologici.

Un miglioramento della capacità discri- minante dei test è stato ottenuto dal nostro gruppo con l'utilizzazione di metodiche di analisi multivariata quali l'analisi di Bayes (2-7).

Attualmente, per una più attendibile valutazione della bilancia simpatico-parasimpatico, il nostro gruppo sta applicando l'analisi spettrale allo studio dell'intervallo R-R, che può consentire la registrazione di alterazioni, anche minime, del controllo del SNV sul sistema cardiovascolare (4). Gli studi effettuati negli ultimi anni su ampie casistiche di pazienti selezionati, utilizzando una batteria di test vegetativi cardiovascolari, hanno dimostrato che una neuropatia parasimpatica, dovuta nella maggior parte dei casi ad una lesione del ramo efferente dell'arco riflesso, si verifica nei pazienti uremici cronici più frequentemente di una lesione del simpatico (8).

Negli uremici cronici in trattamento conservativo è stato osservato un incremento dei valori di noradrenalina plasmatica, una ridotta risposta degli adrenorecettori e spesso una evidente alterazione della attività parasimpatica.

La reale frequenza delle alterazioni riscontrabili a livello strumentale è stata calcolata solo in alcuni studi: nel 14$34 \%$ dei pazienti è presente una alterazione isolata della frequenza cardiaca, che appare invece combinata con una alterazione del controllo simpatico nel 18$26 \%$ dei pazienti (8-11). Spesso queste alterazioni sono subcliniche, ma possono essere causa di ipotensione ortostatica e contribuire agli episodi di ipotensione durante il trattamento dialitico.

$\mathrm{Da}$ un nostro studio eseguito su $35 \mathrm{sog}$ getti uremici cronici in emodialisi periodica è emerso che il $34 \%$ degli esaminati presentava episodi di ipotensione intradialitica ed ortostatica e che gli stessi presentavano altre manifestazioni cliniche suggestive di neuropatia autonomica, quali senso di ripienezza gastrica, ritardo nello svuotamento gastrico, alterazioni della motilità intestinale con diarrea e stipsi, ridotta sudorazione, alterazione del controllo della FC, impotenza (Fig. 6).

Sei soggetti mostravano normale risposta a tutti i test autonomici (17\%), quindici mostravano risposta anomala solo ad un test $(14 \%)$, cinque $(14 \%)$ presentavano un chiaro danno del SNV parasimpatico con risposta anomala a due test, mentre nove (26\%) presentavano un danno combinato del SN parasimpatico e simpatico. Allorché venivano statisticamente para- 


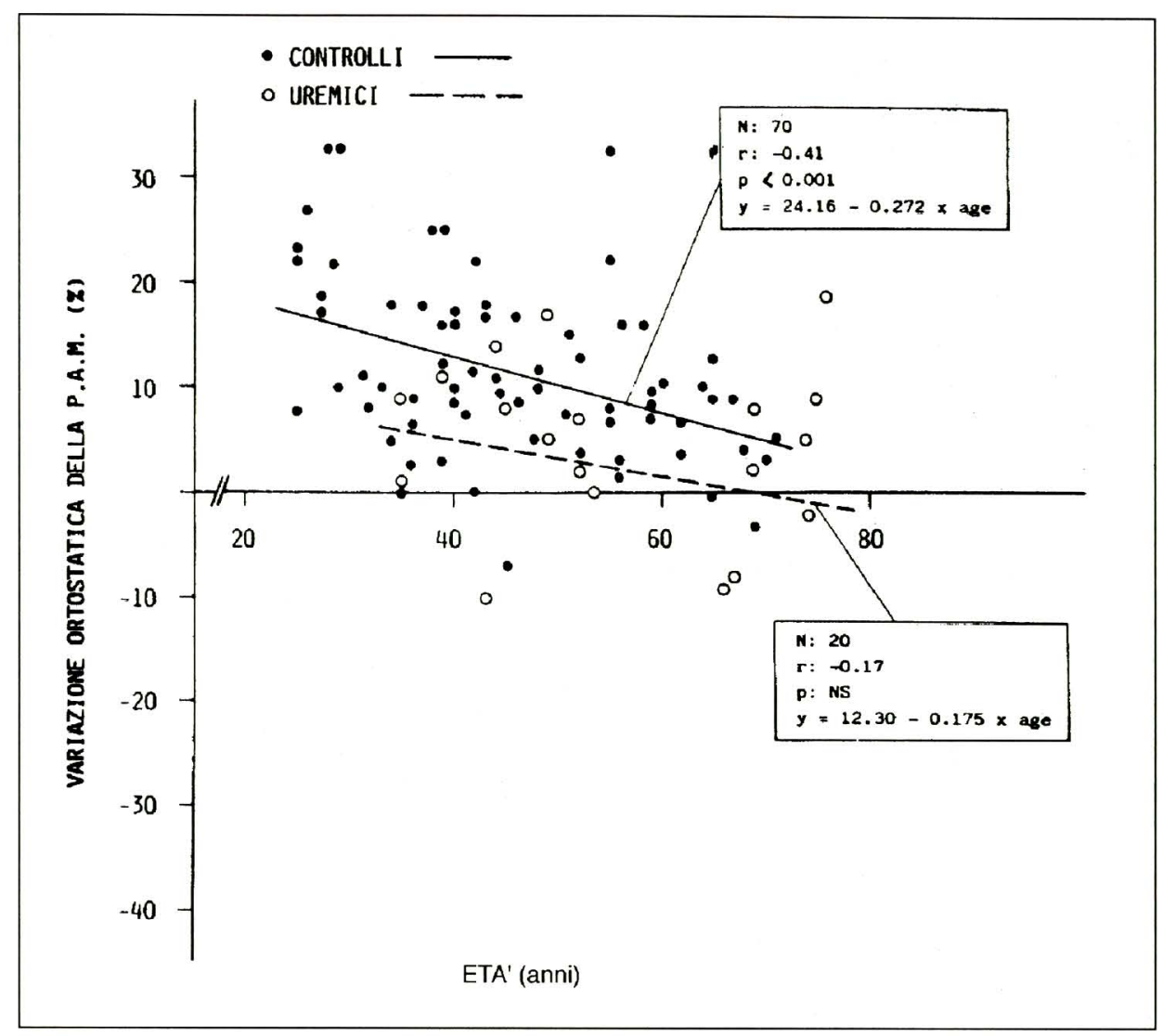

Fig. 7 - Variazioni della FC all' iperpnea (battiti/min) in pazienti d' età geriatrica e in adulti.

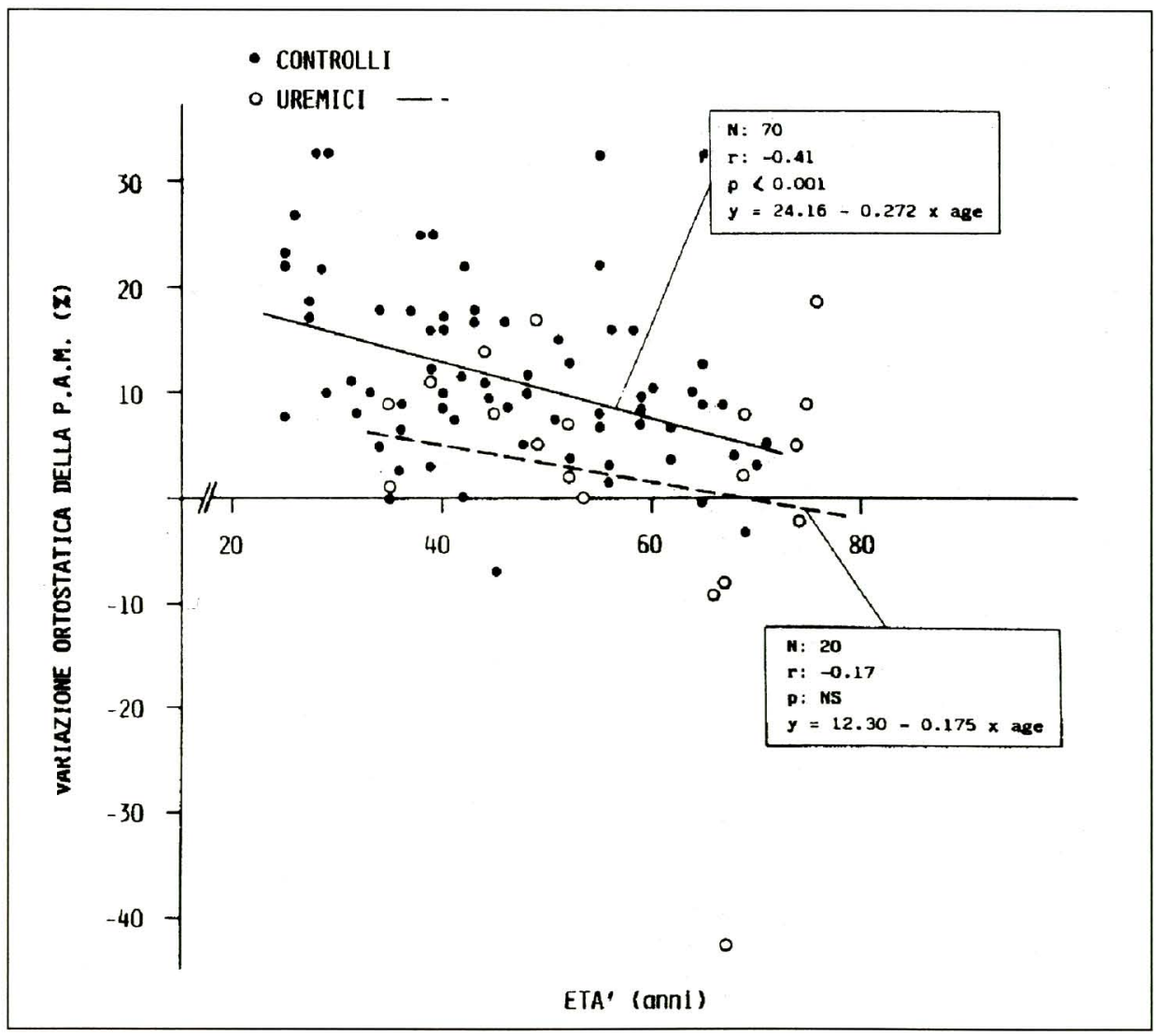

Fig. 8 - Variazione ortostatica della PAM (\%) in pazienti d' età geriatrica e in adulti. gonati il gruppo di soggetti con danno autonomico e quello comprendente gli uremici esenti da tale danno, l'unico parametro significativo risultava essere l'età dialitica.

Un aspetto importante da tenere in considerazione è rappresentato dall'età anagrafica; infatti gli uremici cronici in età geriatrica, nonostante il trattamento emodialitico periodico, presentano un danno parasimpatico e simpatico più grave rispetto ai pazienti di età adulta, il che indica che il loro SNV è più vulnerabili alle "noxae" uremiche (12) (Figg. 7-10).

Un nostro recente studio effettuato mediante un follow-up fino a 56 mesi ha messo in evidenza una estrema variabilità del decorso della funzionalità vegetativa durante il trattamento emodialitico periodico con una chiara tendenza verso un progressivo peggioramento (13).

Altri nostri dati preliminari, relativi ad un follow-up della durata di 96 mesi, suggeriscono la necessità di tempi molto lunghi in emodialisi per ottenere un miglioramento del danno vegetativo ed inoltre l'utilità della bicarbonato-dialisi rispetto a quella con acetato.

Secondo alcuni Autori l'inizio di un trattamento emodialitico cronico è seguito da un miglioramento più o meno marcato della funzionalità vegetativa $(18,19)$. Secondo altri invece non ci sono differenze significative tra pazienti in trattamento conservativo e quelli in emodialisi $(19,20)$ e neanche tra quest'ultimi ed $\mathbf{i}$ pazienti in dialisi peritoneale $(20,21)$.

Nei soggetti uremici cronici trattati con emofiltrazione, dopo riduzione del peso corporeo e del volume plasmatico, al contrario di quanto si verifica in emodialisi, e negli stessi soggetti, non si modificano significativamente la PA media e la $\mathrm{FC}$; a tal proposito è stato possibile riscontrare, dopo emofiltrazione, un aumento dei valori di noradrenalina plasmatica non registrata dopo emodialisi.

Dati di Baldamus et al (16), però, dimostrando una rimozione quantitativa di catecolamine per lo più analoga sia dopo emodialisi che dopo emofiltrazione, suggeriscono una differente risposta del Sistema Nervoso Autonomo ed escludono, come ipotesi etiopatogenetica, la perdita dialitica del neurotrasmettitore.

Dati di Zucchelli et al (16) relativi allo studio della via efferente simpatica valutata mediante esecuzione del cold pressor test sia in HD che in HF, non hanno fornito supporto certo per poter ammet- 


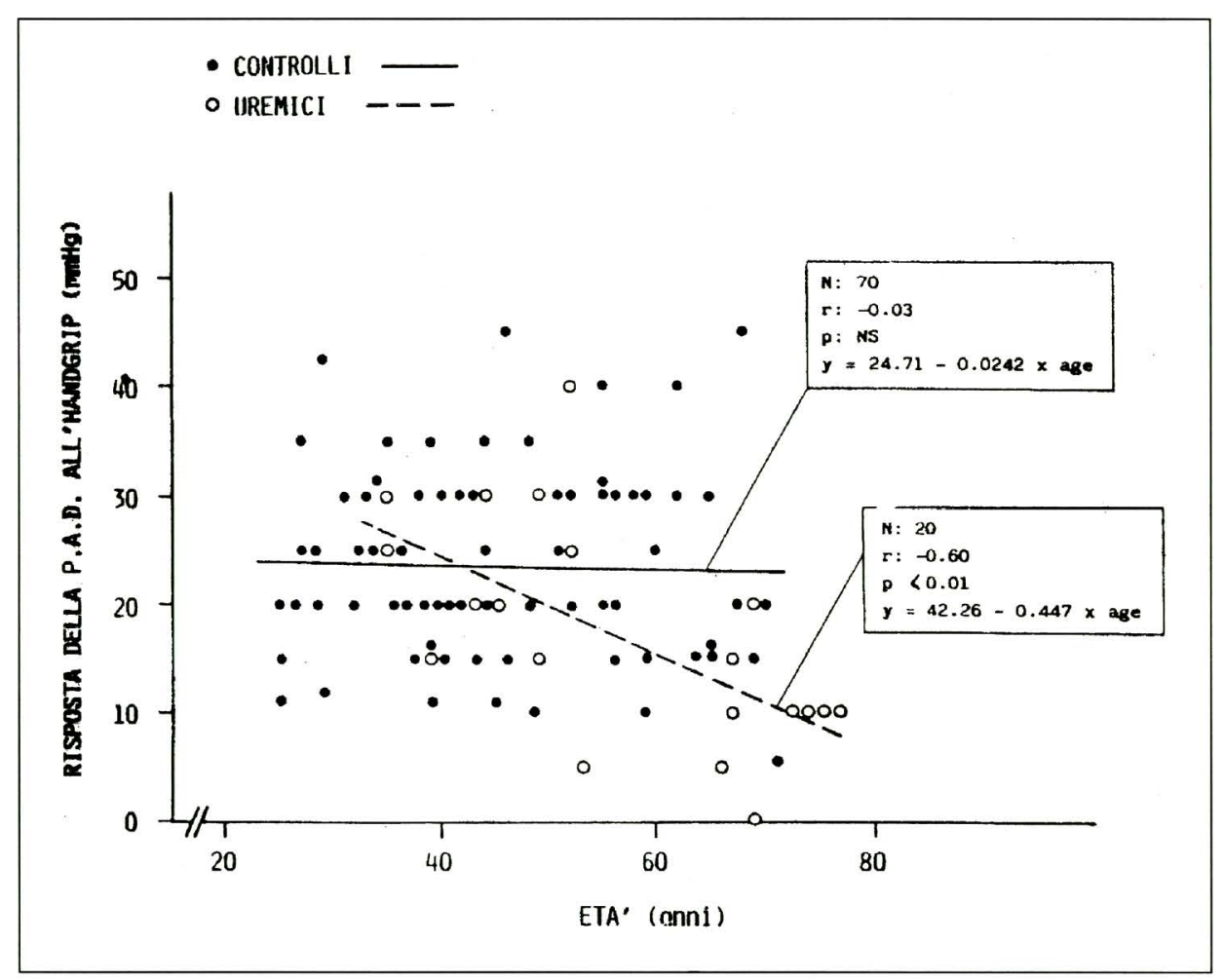

Fig. 9 - Risposta della PAD all' Handgrip ( $\mathrm{mmHg}$ ) in pazienti d' età geriatrica e in adulti. tere una alterazione afferenziale o della risposta recettoriale. Inoltre lo stesso Autore ha potuto dimostrare che il Valsalva ratio è più alto dopo HF che dopo HD e che la funzione barorecettoriale, studiata valutando l'indice di sensibilità barorecettoriale, aumenta dopo $\mathrm{HF}$ mentre si riduce dopo HD.

Il meccanismo patogenetico della neuropatia uremica rimane ancora sconosciuto anche se varie ipotesi sono state avanzate, come per esempio la deficienza vitaminica, l'ischemia, le anomalie del metabolismo del mioinositolo, la perdita di metaboliti utili. L'anemia e la presenza di ipertensione sembrano avere un ruolo patogenetico nella neuropatia vegetativa. Analoga conclusione è stata ottenuta per quanto riguarda il possibile ruolo svolto dalla mancata rimozione di sostanze a medio peso molecolare; infatti non è stato dimostrato alcun effetto sulla funzionalità vegetativa della dialisi peritoneale, che, come è noto, consente a differenza dell'emodialisi, una migliore clearance delle cosiddette mediomolecole (21).

\begin{tabular}{|c|c|c|c|c|c|c|c|}
\hline \multirow[b]{3}{*}{ Test parasimpatici } & \multicolumn{7}{|c|}{ RISULTATI (media \pm DS) OTTENUTI NEI VARI GRUPPI DI STUDIO } \\
\hline & \multicolumn{2}{|c|}{ CONTROLLI } & \multicolumn{2}{|c|}{ UREMICI } & \multicolumn{3}{|c|}{ SIGNIFICATIVITÀ } \\
\hline & $\begin{array}{l}\text { adulti } \\
\text { n. } 11\end{array}$ & $\begin{array}{c}\text { anziani } \\
\text { n. } 9\end{array}$ & $\begin{array}{l}\text { adulti } \\
\text { n. } 11\end{array}$ & $\begin{array}{l}\text { anziani } \\
\text { ก. } 9\end{array}$ & $\begin{array}{c}\text { uremici } \\
\text { adulti/anziani }\end{array}$ & $\begin{array}{c}\text { adulti } \\
\text { contr/uremici }\end{array}$ & $\begin{array}{c}\text { anziani } \\
\text { contr/uremici }\end{array}$ \\
\hline $\begin{array}{l}\text { Variabilità intervallo } \\
\mathrm{R}-\mathrm{R} \text { a riposo (ms) }\end{array}$ & $35.6 \pm 12$ & $24.1 \pm 7.5$ & $21.7 \pm 9.1$ & $21.2 \pm 13.8$ & NS & $p<0.01$ & NS \\
\hline Iperpnea (battiti/min) & $18.1 \pm 5.1$ & $8.4 \pm 3.9$ & $9.3 \pm 6$ & $4 \pm 2.3$ & $p<0.025$ & $p<0.005$ & $p<0.02$ \\
\hline Rapporto di Valsalva & $1.83 \pm 0.38$ & $1.39 \pm 0.12$ & $1.34 \pm 0.27$ & $1.1 \pm 0.13$ & $p<0.05$ & $p<0.005$ & $p<0.001$ \\
\hline Rapporto $30 / 15$ & $1.31 \pm 0.24$ & $1.11=0.09$ & $1.10=0.03$ & $1.06 \pm 0.08$ & NS & $p<0.01$ & NS \\
\hline
\end{tabular}

Test simpatici

\begin{tabular}{lccccccc}
\hline $\begin{array}{l}\text { Variaz. ortostatica } \\
\text { della P.A.M. (\%) }\end{array}$ & $11.4 \pm 4.86$ & $4.44 \pm 4.90$ & $5.81 \pm 7.47 \cdot 2.33 \pm 18.3$ & NS & NS & NS \\
$\begin{array}{l}\text { Contraz. muscolare } \\
\text { isometrica (mmHg) }\end{array}$ & $23.6 \pm 10.9$ & $18.3 \pm 13.2$ & $23.1 \pm 9.5$ & $10 \pm 5.5$ & $p<0.005$ & NS & NS
\end{tabular}


È stato anche suggerito un ruolo etiopatogenetico del paratormone che sperimentalmente ha mostrato esercitare effetti neurotossici $(15,29)$.

Sulla base del riscontro di elevati livelli plasmatici di metenkefalina negli uremici cronici, è stato suggerito un ruolo patogenetico degli oppioidi endogeni.

Questa ipotesi viene sostenuta dall'osservazione che il naloxone, un antagonista degli oppiacei, è in grado di indurre negli uremici un aumento significativo del rapporto di Valsalva attraverso una aumentata attivazione parasimpatica durante la fase IV della manovra (1). È stato infine ipotizzato che la neuropatia vegetativa uremica possa essere imputata non solo alla lesione di vie nervose periferiche ma anche a lesioni di importanti nuclei di controllo vegetativo a livello del Sistema Nervoso Centrale $(10,11)$.

\section{BIBLIOGRAFIA}

1. Vita G, Princi P, Calabrò R, et al. Cardiovascular reflex tests. Assessment of age-adjusted normal range. J Neurol Sci 1986; 75: 263-74.

2. Vita G, Princi P, Messina C Multivariate analysis of cardiovascular reflexes applied to the diagnosis of autonomic neuropathy. J Neurol 1991; 238: 251-5.

3. Vita G, Princi P, Savica V, et al. Uremic autonomic dysfunction evaluated by pattern recognition analysis. Clin Nephrol 1991; 36: 290-3.

4. Bellavere F, Balzani I, De Masi G, et al. Power spectral analysis of heart-rate variations improves assessment of diabetic cardiac autonomic neuropathy. Diabetes 1992; 41: 633-40.

5. Savica V, Vita G, Princi P, et al. Analisi multivariata dei riflessi vegetativi cardiovascolari nell'uremia. In: M. Timio and S. Venanzi eds. Il Cuore nelle Nefropatie e nella Dialisi. Bologna: Monduzzi Editore, 1989; 311-4.

6. Vita G, Dattola R, Calabrò R, et al. Comparative analysis of autonomic and somatic dysfunction in chronic uraemia. Eur Neurol 1988; 28: 335-40.

7. Vita G, Dattola R, Puglisi RM, et al. Autonomic nervous system dysfunction in chronic uraemics on haemodialysis. Funct Neurol 1989; 4: 195-7.

8. Vita G, Messina C, Savica V, Bellinghieri G. Uraemic autonomic neuropathy. J Auton Nerv Syst 1990; 30: S179-84.

9. Malik S, Wimney RJ, Ewing DJ. Chronic renal failure and cardiovascular autonomic function. Nephron 1986; 43: 191-5.

10. Vita G, Dattola R, Calabrò R, et al. Comparative analysis of autonomic and somatic dysfunction in chronic uraemia. Eur Neurol 1988; 28: 335-40.

11. Vita G, Dattola R, Puglisi RM, et al. Autonomic nervous system dysfunction in chronic uraemics on haemodialysis. Funct Neurol 1989; 4: 195-7.

12. Vita G, Savica V, Marabello L, et al. Occurrence of uraemic autonomic dysfunction in relation to age. Acta Neurol Scand 1987; 76: 46872.

13. Vita G, Savica V, Puglisi RM, Marabello L, Bellinghieri G, Messina C. The course of autonomic neural function in chronic uraemic patients during haemodialysis treatment. Nephrol Dial Transplant 1992; 7: 1022-5.

14. Bach C, Iaina A, Eliahou $\mathrm{HE}$ Autonomic nervous system disturbance in patients on chronic hemodialysis. Isr J Med Sci 1979; 15: 761-4.

15. Solders G, Persson A, Gutierrez A. Autonomic dysfunction in non-diabetic terminal uraemia. Acta Neurol Scand 1985; 71: 321-7.

16. Zucchelli P. Fattori di instabilità cardiocircolatoria in emodialisi. In: M. Timio, S. Venanzi, eds. Cuore e Rene Wichtig Editore Milano 1989; 27-32.

17. Campese VM, Romoff MS, Levitan D, Lane K, Massry SG. Mechanisms of autonomic nervous system dysfunction in uremia. Kidney Int 1981; 20: 246-53.

18. Heidbreder E, Schafferhans K, Heidland A. Autonomic neuropathy in chronic renal insufficiency: comparative analysis of diabetic and nondiabetic patients. Nephron 1985; 41: 50-6.

19. Malik S, Winney RJ, Ewing DJ. Chronic renal failure and cardiovascular autonomic function. Nephron 1986; 43: 191-5.

20. Rockel A, Hennemann $\mathrm{H}$, Sternagel-Haase A, Heidland A. Uraemic sympathetic neuropathy af- 
ter haemodialysis and transplantation. Eur J Clin Invest 1979; 9: 23-7.

21. Mallamaci F, Zoccali C, Ciccarelli M, Briggs JD. Autonomic function in uremic patients treated by hemodialysis or CAPD and in transplant patients. Clin Nephrol 1986; $25: 175-80$.

22. Solders G, Persson A, Wilczek H. Autonomic system dysfunction and polyneuropathy in nondiabetic uremia. A one-year follow-up study after renal transplantation. Transplantation 1986; 41: 616-9.

23. Agarwal A, Anand IS, Sakhuja V, Chugh KS. Effect of dialysis and renal transplantation on autonomic dysfunction in chronic renal failure. Kidney Int 1991; 40: 489-95.

24. Heidbreder E, Schafferhans K, Gotz R, Heidland A. Autonomic neuropathy in diabetic nephropathy: effect of regular dialysis treatment and simultaneous transplantation of kidney and pancreas. Contrib Nephrol 1989; 73: 112-26.

25. Solders G, Wilczek H, Gunnarsson R, Tyden G, Persson A, Groth CG. Effects of combined pancreatic and renal transplantation on diabetic neuropathy: a two-year follow-up study. Lancet 1987; 2: 1232-4.

26. Gaber AO, Cardoso S, Pearson $S$, et al. Improvement in autonomic function following combined pancreas-kidney transplantation. Transplant Proc 1991; 23: 1660-2.

27. Solders G, Tyden G, Persson A, Groth CG. Improvement of nerve conduction in diabetic neuropathy: a follow-up study $4 \mathrm{yr}$ after combined pancreatic and renal transplantation. Diabetes 1992; 41: 946-51.

28. Boucek P, Bartos V, Vanek I, Hyza Z, Skibova J. Diabetic autonomic neuropathy after pancreas and kidney transplantation. Diabetologia 1991, 34 (Suppl. 1): S121-4.

29. Vita G, Savica V, Calabrò R, et al. Uremic vagal neuropathy: has parathyroid hormone a pathogenetic role. Funct Neurol 1986; 1: 253-9. 